$$
\text { NTiS HC \$4.25 }
$$

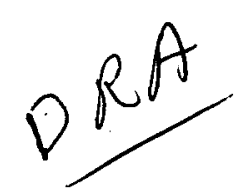

\title{
STELLAR EVOLUTION AT HIGH MASS BASED ON THE LEDOUX CRITERION FOR CONVECTION
}

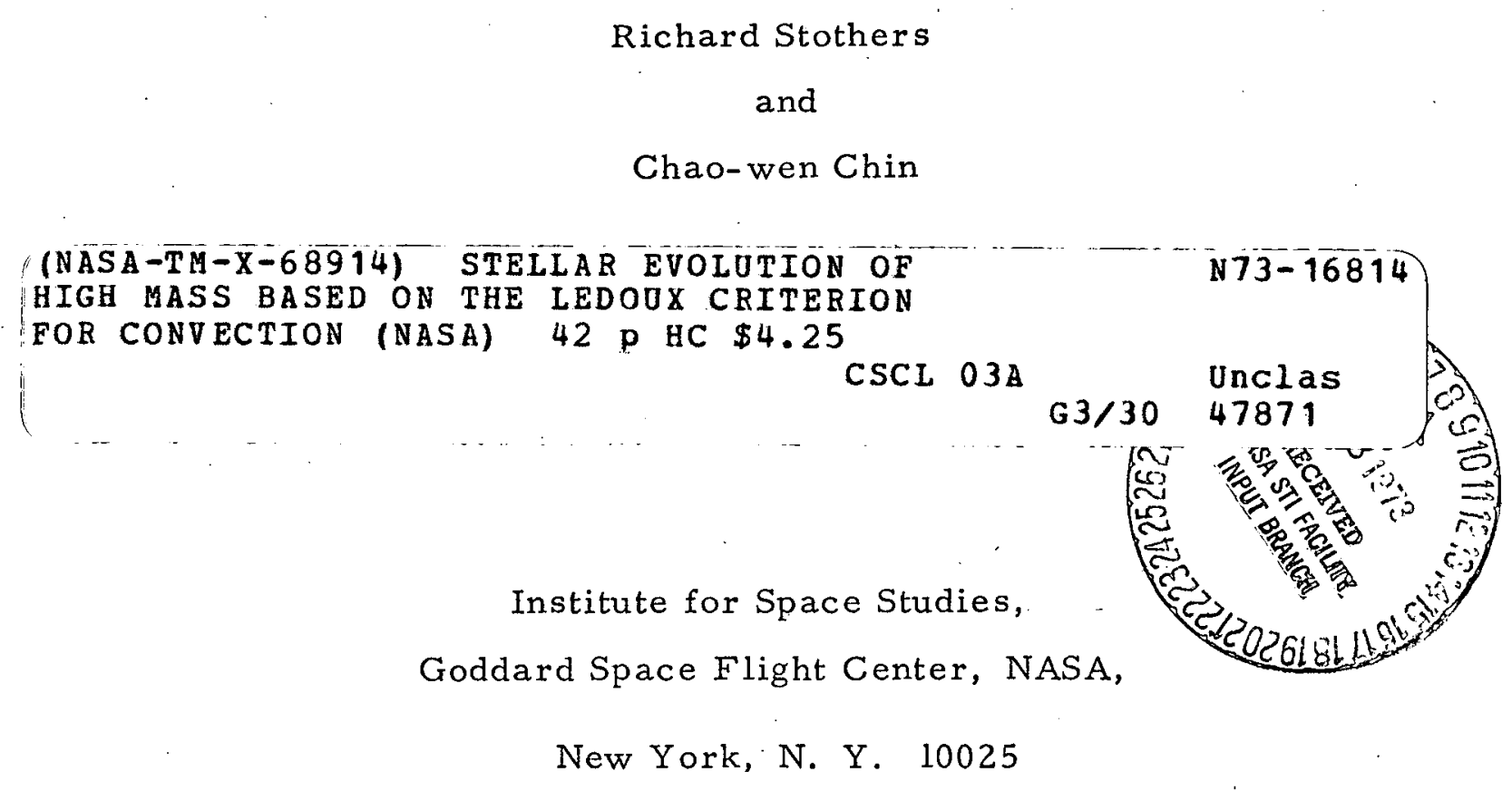




\section{ABSTRACT}

Theoretical evolutionary sequences of models for stars of 15 and $30 \mathrm{M}_{\odot}$ have been computed from the zero-age main sequence to the end of core helium burning, with the use of the Ledoux criterion for convective instability. The sensitivity of the models to the most important physical input parameters has been tested. During the earliest stages of core helium depletion, the envelope rapidly expands into the red-supergiant configuration because the hydrogen profile in the intermediate zone remains essentially unaltered by local convection. At $15 \mathrm{M}_{\odot}$, a blue loop on the $\mathrm{H}-\mathrm{R}$ diagram ensues if the initial metals abundance, initial helium abundance, or ${ }^{12} \mathrm{C}+\alpha$ reaction rate is sufficiently large, or if the $3 \alpha$ reaction rate is sufficiently small. These quantities affect, respectively, the opacity (and hence depth) of the base of the outer convection zone, the mass of the core, and the thermal properties of the core. The blue loop occurs abruptly and fully developed when the "critical" value of any of these quantities is exceeded, and the effective temperature range and fraction of the lifetime of core helium burning during the slow phase of the blue loop vary surprisingly little. For the recently revised $3 \alpha$ reaction rate, the blue loop always forms. At $30 \mathrm{M}_{\odot}$, no blue loop occurs for any reasonable 
set of input parameters - unless the temporary convective intermediate zone (ignored in the present work) extends deeper than does the outer convection zone. For both masses, all passages of the Hertzsprung gap occur on the thermal time scale of the envelope. 


\section{INTRODUCTION}

The internal structure of stars evolving on the upper main sequence is still poorly known (Stothers 1970). Two persistent problems are (1) the correct criterion to use for convective instability in a region that contains a gradient of mean molecular weight and (2) the nature of any resulting convective motions. The two possible criteria for convective instability are the Ledoux criterion (which includes the gradient of mean molecular weight) and the Schwarzschild criterion (which does not). Even were the correct criterion known, it is not certain what the final state of the convective motions would be. At present, the most popular solutions for the internal structure are based on a shrinking convective core, in which hydrogen is continually being depleted; an intermediate zone of varying hydrogen-helium content; and an ' outer radiative (or mostly radiative) envelope of zero-age composition. Although the differences among the various solutions have only slight effect on the main sequence phase, the details of the further evolution are very sensitive to them. This is particularly true in regard to the direction of evolution (including the occurrence of "loops") on the H-R diagram. 
1

Few published evolutionary sequences for massive stars have been based (implicitly or explicitly) on the Ledoux criterion, and have been carried through to the end of core helium burning; those available are listed in table 1, where earlier sequences are omitted in which the sole opacity source is electron scattering (see Stothers and Chin 1968 and references therein). In the present paper, we propose to examine in more detail the evolution of massive stars based on the Ledoux criterion.

II. EVOLUTIONARY SEQUENCES BASED ON THE LEDOUX CRITERION

Theoretical evolutionary sequences for stars with masses of 15 . and $30 \mathrm{M}_{\odot}$ have been calculated by use of an automatic computer program whose basic ingredients are given in the Appendix. Table 2 lists the following input parameters for each sequence: $\mathrm{x}_{e}$, the initial hydrogen abundance by mass; $\mathrm{Z}_{e}$, the initial metals abundance by mass; ${ }^{2} \alpha$, the reduced $\alpha$-particle width of the $7.12-\mathrm{MeV}$ level in ${ }^{16} \mathrm{O} ; \epsilon_{3 \alpha}$, the rate of nuclear energy generation from the $3 \alpha$ process; $\alpha$, the ratio of convective mixing length to density scale height; and $x$, the (low-temperature) opacity. The recently revised $3 a$ rate (Austin, Trentelman, and Kashy 1971) will be called here the "new" rate, whereas the previously used rate (Clayton 1968) will be called the "old" rate. Our basic set of opacities (Appendix) will be referred 
to as the "unmodified" opacities, while the same set multiplied by a temperature-dependent increment to the low-temperature $<10000$ ${ }^{\circ} \mathrm{K}$ ) values will be referred to as the "modified" opacities. Magnetic fields and mass loss have been ignored in our models as being probably unimportant (Stothers 1972b, c a and references therein); rotation has also been ignored although it is undoubtedly of some importance. Each sequence has been evolved from the initial stage of core hydrogen burning. (ZAMS) to the end of core helium burning. -

Although the general features of evolution in massive stars have already been described elsewhere (e. g., Stothers 1966; Stothers and Chin 1968), we shall here summarize the salient characteristics of our models because (1) they differ in a few significant aspects from our earlier models, (2) they provide a framework for our subsequent discussion of changes in the input parameters, and (3) they are relevant to our discussion of the relative amounts of time that a massive star spends burning core heium in various parts of the $H-R$ diagram. Three representative evolutionary tracks for each mass, based on three different initial chemical compositions, are shown in figure 1. The interior distributions of hydrogen at several stages of evolution for two of these initial chemical compositions are shown in figure 2 for $15 M_{\odot}$. 
Beginning at the ZAMS, the main phase of core hydrogen burning is characterized by an extensive convective core, which depletes its hydrogen, recedes monotonically in time, and leaves behind a convectively stable intermediate zone of varying hydrogenhelium content. The slowly expanding outer envelope is also convectively stable, except for a small region just above the zero-age core boundary in $30 \mathrm{M}_{\odot}$ where convection develops during the late stages of core hydrogen burning. 1

$1_{\text {If the adopted opacity source is pure Thomson }}$ scattering, convection develops just outside the zero-age core for masses as low as $10 \mathrm{M}_{\odot}$ (Schwarzschild and Härm 1958). Furthermore, if convective overshooting into the immediately underlying radiative layers is important, a small semiconvective zone will develop there (Sakashita and Hayashi 1961; Stothers 1963).

When the central hydrogen content drops to $\mathrm{X}_{c}=0.030 \pm 0.005$ (see also Stothers 1964), the star undergoes an overall contraction; the hydrogen immediately surrounding the convective core heats up and a hydrogen-burning shell is formed..

After the complete exhaustion of hydrogen at the center, the core undergoes a rapid contraction, while the envelope re-expands 
vigorously. Convection at the center eventually vanishes during this phase if the stellar mass is less than $\sim 30 \mathrm{M}_{\odot}^{2}$ (see also

2 More exactly, if the core mass is less than
$\sim 10 \mathrm{M}_{\odot}$. The corresponding mass for an ideal core
characterized by a pure helium composition, pure
Thomson scattering opacity, and purely homologous
contraction is $8 \mathrm{M}_{\odot}$ (Tanaka and Sakashita 1964).

Stothers 1964). Ignition of central helium causes the redevelopment of central convection, and helium burning finally stabilizes the contracting core just before, or during, the crossing of the Hertzsprung gap. However, the envelope keeps on expanding (on a thermal time scale) until the tip of the red-supergiant branch is reached. By this time, some 3 percent of the core helium is consumed.

The region immediately below the zero-age core boundary becomes convectively unstable during the crossing of the Hertzsprung gap. Any temporary convective modifications of this region (ignored in our models but included by Stothers 1966) are soon obliterated by the deep convective envelope which extends down from the surface - certainly this happens at $15 \mathrm{M}_{\odot}$ although possibly not at $30 \mathrm{M}_{\odot}$ 
(see Chiosi and Summa 1970; Robertson 1972). The maximum depth achieved by the convective envelope occurs when the star is at or near the tip of the red-supergiant branch. There the surface hydrogen abundance is reduced by 1 to 10 percent (depending on the mass and initial chemical composition) because convection penetrates to the helium-rich intermediate layers; simultaneously, the surface ${ }^{12} \mathrm{C} /{ }^{14} \mathrm{~N}$ ratio is also reduced (see Iben 1964) although we have not determined the amount here.

Subsequent evolution down the red-supergiant branch takes place slowly on a nuclear time scale. The descent is halted prematurely if the envelope becomes again thermally unstable and contracts rapidly to the blue-supergiant configuration; thereupon, depletion of core helium proceeds within a thermally stable blue supergiant. Sometimes a small S-bend, or stillstand, occurs at the blue tip of the evolutionary loop, before the envelope re-expands vigorously during the last stages of core helium burning, ultimately to cross again the Hertzsprung gap on a thermal time scale (usually after the exhaustion of central helium). However, if the star's envelope on the red-supergiant branch remains thermally stable throughout the phase of core helium burning, the descent of the red branch continues to fainter luminosities, down to a precarious point 
where the star may "hover" for a time before making a final re-ascent. Envelope convection at this minimum luminosity is found to cover a much smaller mass fraction at $15 \mathrm{M}_{\odot}$ than at $30 \mathrm{M}_{\odot}$.

For ${ }_{\alpha}^{2}=0$ and $\theta_{\alpha}^{2}=1$, the final chemical composition of the helium-exhausted convective core is always pure carbon and pure oxygen, respectively. For $\theta_{\alpha}^{2}=0.1$, the final carbon abundance is $\mathrm{X}_{C}=0.15 \pm 0.05$ for the new 30 rate for $\mathrm{X}_{C}=0.35 \pm 0.05$ for the old $3 \alpha$ rate).

The last seven columns of table 2 give the following data: $\log T_{e}$, logarithm of the effective temperature at the tip of the blue loop during core helium burning; $\tau_{H}$, lifeti me of core hydrogen burning (until $\mathrm{X}_{\mathrm{c}}=0$ ); $\tau_{\mathrm{He}} / \tau_{\mathrm{H}}$ ' ratio of the lifetime of core helium burning (until $Y_{c}=0$ ) to that of core hydrogen burning; $\tau_{b}(1) / \tau{ } \mathrm{He}^{\text {, }}$ $\tau_{b}(2) / \tau_{H e}$, fraction of time spent in the first (1) and second (2) blue-supergiant configurations $\left(\log \mathrm{T}_{\mathrm{e}} \geqslant 4.1\right)$ during core helium burning; $\tau_{y}(1) / \tau_{H e}, \tau_{y}(2) / \tau_{H e}$, fraction of time spent in the first (1) and second (2) yellow-supergiant configurations $\left(3.6 \leqslant \log \mathrm{T}_{\mathrm{e}}\right.$ \$4. 1) during core helium burning. The last significant figures given for the blue and yellow lifetimes are rather uncertain. because of the uncertainty of the effective temperature boundaries. 
Generally speaking, our evolutionary sequences are in good qualitative agreement with previously published sequences based on the Ledoux criterion (table 1). Our specifically new results, and some differences of interpretation, are discussed below.

\section{INV்ESTIGATION OF $1.5 \mathrm{M}_{\odot}$}

Our most extensive set of model sequences, designed to test the effect of changing various physical parameters, has been carried out for $15 \mathrm{M}_{\odot} \cdot$ Properties of the sequences, other than the blue loops, which are discussed below, will be analyzed next in terms of these physical parameters.

\section{a) Nuclear Reaction Rates}

Hydrogen burning via the CN cycle completely outweighs the pp chain, which has been ignored in our models. However, possible errors in the ${ }^{14} \mathrm{~N}+\mathrm{p}$ reaction rate and, more significantly, in the assumed equilibrium abundance of ${ }^{14} \mathrm{~N}$ have been assessed by considering the two cases $x_{C N}=\frac{1}{3} z_{e}$ and $x_{C N O}=\frac{2}{3} Z_{e}$, which are introduced as the ${ }^{14} \mathrm{~N}$ abundance in the reaction rate. Virtually no difference in results is found between our two cases (table 2) except for a slight lengthening of lifetimes and a slight brightening of the tip of the red-supergiant branch due to enhanced 
shell burning and to an enlarged core mass, when ${ }^{14} \mathrm{~N}$ is more abundant.

Nitrogen burning via $\alpha$ capture has been ignored as an energy source in all of our models since the revised reaction rate for ${ }^{14} \mathrm{~N}+\alpha$ implies an unfavorable competition with $3 \alpha .{ }^{3}$ However, the

$$
{ }^{3} \text { Even with the old rate, }{ }^{14} N+\alpha \text { scarcely affected }
$$

the model sequences (Iben 1966a, b; Simpson 1971).

revised reaction rate for $3 \alpha$ causes helium to burn at a hotter temperature than before, and so produces a somewhat brighter tip of the red-supergiant branch and a deeper convective envelope; the elevated temperature also means that carbon is depleted faster via ${ }^{12} \mathrm{C}+\alpha$. At the present time, ${ }^{12} \mathrm{C}+\alpha$ can be considered the only important reaction whose rate is still uncertain. Model sequences based on the choices of $\frac{\theta^{2}}{\alpha}=0,0.1$, and 1 for this reaction show than an increase in $\rho_{\alpha}^{2}$ (by augmenting the formation of oxygen as well as the size of the convective core) prolongs the lifetime of helium burning.

\section{b) Convective Envelope Parameters}

Our sequences are found to be not greatly affected by changes 
in the physical parameters $\alpha$ and $x$ pertaining to layers above the base of the outer convection zone, because the location of the base is itself not much affected. This result has been obtained earlier (Stothers 1972a), and is due to the fact that, in massive stars, the superadiabatic region near the surface covers only a small fraction of the total mass. However, the surface temperature can be adjusted to agree with observations by selecting an appropriate value of $\alpha$ or of low-temperature opacity (Henyey, Vardya, and Bodenheimer 1965; Stothers 1972a), although no physical significance should be attached to such arbitrary selections, which merely mask the uncertainties in the mixing-length theory.

\section{c) Initial Chemical Composition and Opacity}

The initial hydrogen (and helium) content of the star influences its structure primarily through the mean molecular weight (and, to a lesser extent, through the opacityl. As can be shown by simple similarity arguments, an increase in $\mathrm{X}_{\mathrm{e}}$ reduces the luminosity and mass fractions of composition boundaries (including the base of the outer convective envelope), and therefore increases the lifetime of the various phases. However, as shown by figure 3 , the mass separations between composition boundaries are nearly independent of $X_{e}$, as is the ratio of the hydrogen-shell luminosity 
to the total luminosity (at a given stage of evolution). But the fraction of the total stellar lifetime spent burning core helium is slightly less for large values of $\mathrm{X}_{e}$ because less oxygen is formed in smaller cores.

The changes induced by an increase in $Z_{e}$ are quite similar to those induced by an increase in $X_{e}$, with the main exception that the convective envelope penetrates to a disproportionately deeper level (see figure 3). Such a change can be induced also by an increase in the opacity coefficient alone, but this is equivalent to an increase in $Z_{e}$ since the latter quantity influences the structure of the star primarily through the opacity. The depth of the convective envelope is determined, in part, by the opacity near its base, where the temperature (for a deep envelope) is normally $\sim 10^{6}{ }^{\circ} \mathrm{K}$; if the opacity is large at this temperature, the radiative temperature gradient will remain steep to a greater depth than otherwise.

\section{d) The Blue Loops}

The chief feature which differentiates the various sequences on the $\mathrm{H}-\mathrm{R}$ diagram is the appearance, or nonappearance, of a long blue loop during core helium burning. The precise cause of the loops is still obscure. The "mirror principle" (Hayashi, Hōshi, and Sugimoto 1962; Hofmeister, Kippenhahn, and Weigert 1964), 
which is a phenomenological description (not always valid) of what happens, has been analyzed in a preliminary way by Stein (1966). The roles of $\mathrm{x}_{\mathrm{e}}$, of core mass, of opacity, and of hydgrogen profile in and above the shell have been recognized in our previous study (Stothers and Chin 1968), while Hofmeister (1967) has emphasized the importance of $Z_{e}$. Further studies (Fricke, Stobie, and Strittmatter 1971; Lauterborn, Refsdal and Roth 1971; Barbaro, Chiosi, and Nobili 1971; Dallaporta 1971; Fricke and Strittmatter 1972) have confirmed and greatly amplified the earlier work, and it now seems that such additional features as numerical accuracy in the computations (Paczynski 1970b) and axial rotäition (Kippenhahı, : Meyer-Hofmeister, and Thomas 1970; Meyer-Hofmeister 1972) are also important in determining the loops.

Whether or not a blue loop occurs seems to depend on the existence of multiple solutions of the static stellar configuration for a given run of chemical composition (Paczynski 1970b;

Lauterborn et al. 1971; Kozlowski 1971). ${ }^{4}$ It has further been

${ }^{4}$ Such a multiplicity of solutions was already found among (purely) red-supergiant models for more highly, evolved states (Stothers and Chin 1969). 
shown that initiation of the loop for a massive star takes place on the thermal time scale of the envelope. We have found that the closer a sequence is to a "critical" sequence (one which barely develops a blue loop), the more likely to occur, and the more pronounced, is a thermal fluctuation of the envelope just around the luminosity minimum before the star goes either over to the blue tip or back up to the red tip. To investigate the possibility of thermal instability of the hydrogen-burning shell in connection with the loop phenomenon, we have recalculated parts of our sequence $15-P$ using very small time steps, but have found that the shell always remains thermally, stable (as expected from our analogous study of the earlier stages of evolution [Stothers and Chin 1972]). However, the tendency toward thermal instability in the shell is greatest during the initial phase of the loop. Since the envelope contracts very rapidly at this time, the shell heats up faster than it is cooled by its expansion; therefore it temporarily spreads over a greater mass fraction, and its luminosity rises. This relieves some of the weight off the core, so that the central pressure, and hence the central temperatire and the convective-core boundary, initially diminish a little. Thus, there is feedback from the envelope to the core, and the "isolated star" approximation for the core structure is evidently only valid for quasi-static stages of evolution. 
Although we are unable to give a precise physical explanation for the occurrence of the blue loops, we can, at least, point out four factors which lead to their development: (1) a small value of $\mathrm{X}_{\mathrm{e}}$, (2) a large value of $\mathrm{Z}_{\mathrm{e}}$ (or of interior opacity), (3) a large value of the ${ }^{12} \mathrm{C}+\alpha$ reaction rate, and (4) a small value of the $3 \alpha$ reaction rate. Moreover, a blue loop, when it occurs, does so abruptly for a small change in one of the four parameters, and always appears fully developed on the $\mathrm{H}-\mathrm{R}$ diagram.

Interestingly enough, core mass alone (as fixed by $x_{e}$ ) can determine whether or not a blue loop occurs. Our sequences for $15 \mathrm{M}_{\odot}$ with $\mathrm{Z}_{\mathrm{e}}=0.021, \theta_{\alpha}^{2}=0.1$, and the old $3 \alpha$ rate, have a loop only if $x_{e} \leqslant 0.64$. But, regardless of $x_{e}$, they all contain the same hydrogen gradient in and above the burning shell (at a given stage of evolution), and, in all cases, the shell never makes contact with the hydrogen discontinuity that is formed by the outer convection zone (see figures 2 and 3). The effectiveness of core mass can also be seen in our previously published sequence for $15 \mathrm{M}_{\odot}$ (Stothers and Chin 1968), which initiated a loop at the same core mass $(q \approx 0.32)$ as do our present sequences - even though the hydrogen profile in the shell was very different.

In contrast to the sequences with low $Z_{e}$, our sequences for 
$15 \mathrm{M}_{\odot}$ with $\mathrm{Z}_{\mathrm{e}}=0.044$ have relatively deep convective envelopes. Before the end of core helium burning, the hydrogen-burning shell reaches the hydrogen discontinuity in all cases (see figures 2 and 3), and this produces a long blue loop (see also Stothers and Chin 1968; Robertson 1972). It appears likely that a difference in $\mathrm{Z}_{\mathrm{e}}$ can also. explain why the sequence for $20 \mathrm{M}_{\odot}$ calculated by Chiosi and Summa has a blue loop whereas Robertson's sequence, for very nearly the same value of $X_{e}$, does not (see table 1). Paczynski's adopted metals abundance, $z_{e}=0.03$, used in his sequence for $15 \mathrm{M}_{\odot}$, is "marginal" in the present context, and this may explain why, in two preliminary trial sequences, he obtained a blue loop in one case and no blue loop in the second case (significantly, the loop occurred at almost the end of core helium burning). Our present sequence 15-U spends less time in the blue loop than does Robertson's analogous sequence, because his adopted opacities were larger, resulting in a deeper convective envelope at the tip of the red-supergiant branch and hence an earlier conjunction of the hydrogenburning shell with the hydrogen discontinuity. The variation between Hofmeister's two sequences for $9 \mathrm{M}_{\odot}$ is probally due to the difference in $X_{e}$, although the difference in $Z_{e}$ may be important if the convective envelope in her sequence with large $Z_{e}$ is sufficiently deep. 
Finally, if $\theta_{\alpha}^{2}=1$ and the old $3 \alpha$ rate are adopted, our sequences for $15 \mathrm{M}_{\odot}$ with $Z_{e}=0.021$ exhibit a blue loop whenever $X_{e} \leqslant 0.70$ (to be compared with the much smaller limit on $\mathrm{X}_{\mathrm{e}}$ for the corresponding sequences with $\left.\theta_{\alpha}^{2}=0.1\right)$. But, if the new $3 \alpha$ rate is adopted, all the sequences for $15 \mathrm{M}_{\odot}$ have a blue loop, regardless of the adopted values for the other parameters. Since the changes in the rates of the ${ }^{12} \mathrm{C}+\alpha$ and $3 \alpha$ reactions do not alter the mass of the core or the hydrogen gradient in the envelope, the changes of the thermal properties (alone) of the core are, clearly, the determining factor. However, in the case of the new $3 \alpha$ rate, the slightly deeper location of the hydrogen discontinuity does, for some sequences, assist in promoting the blue loop.

\section{INVESTIGATION OF $30 \mathrm{M}$}

All of our evolutionary sequences of $30 \mathrm{M}_{\odot}$ remain on the redsupergiant branch during core helium burning. There are two major reasons for this. First, the hydrogen gradient, $d X / d M(r)$, in the intermediate zone is very shallow at high mass since the nondimensional gradient $\mathrm{dX} / \mathrm{dq}$ is nearly independent of mass. Second, the outer convection zone does not penetrate very deeply into the star (figure 4), in contradiction to our earlier prediction (Stothers and Chin 1968). Although it is true that convection extends down 
well into the intermediate zone just as it does for lower masses, 5

5

${ }^{5}$ For some reason, the outer convection zone did not reach the intermediate zone in Simpson's (1971) sequence for $30 \mathrm{M}_{\odot}$ (see his figure $5 \underline{a}$ ).

this zone is displaced farther outward in mass fraction at higher masses, and, because of the generally shorter time scale, the hydrogen-burning shell does not advance as far as it does at lower masses, with the result that it fails to reach the hydrogen discontinuity. An earlier sequence calculated by Simon and Stothers (1970) for $\left(X_{e}, Z_{e}\right)$ $=(0.33,0.03)$ did have a blue loop (without the aid of a suddenly encountered hydrogen discontinuity), so that the "critical" hydrogen abundance for $30 \mathrm{M}_{\odot}$ probably lies somewhere between $\mathrm{X}_{\mathrm{e}}=0.33$ and 0.55. The present sequences for $30 \mathrm{M}_{\odot}$ are subject to far less uncertainty about the opacity at temperatures near $10^{6}{ }^{\circ} \mathrm{K}$ than are the sequences at lower mass, due to the predominance of electron scattering at high mass. To check this point, we have computed a special sequence for $30 \mathrm{M}_{\odot}$ with all input parameters favorable to the development of a blue loop: $X_{e}=0.602, z_{e}=0.044, \theta_{\alpha}^{2}=1$, the new $3 \alpha$ rate, and the absorption part of the opacity multiplied by a factor $\max \{1,3-|6-\log T|\}$ (inspired by the opacity calculations of Carson, Mayers, and Stibbs 1968). The sequence remained red. 
Nevertheless, our neglect (and Simpson's neglect) of semiconvective and convective mixing in the intermediate zone may be serious for masses heavier than $15 \mathrm{M}_{\odot}(\S I I)$. It is possible that a convective intermediate zone might extend to a greater depth than that reached by the outer convection zone, and hence induce a blue phase when the hydrogen-burning shell reaches the hydrogen discontinuity. Further studies are necessary to decide on this question.

Bisnovatyi-Kogan and Nadezhin (1972) obtained results for $30 \mathrm{M}_{\odot}$ that are qualitatively different from Simpson's and our results. Since these authors were unable to obtain convergent, static solutions for the atomspheres of yellow-supergiant models and also found a large density inversion near the surface of their last static models, they postulated a steadily outflowing atmosphere as the proper boundary condition for the next stages, and derived a mass loss rate of $0.5 \mathrm{M}_{\odot}$ per year. We object to their solution on three counts. First of all, our probably more accurate treatment of the static atmospheres (see Appendix) always led to consistent, convergent solutions; more importantly, Osmer (1972) also obtained . convergent solutions for much more detailed model atmospheres. (Both Osmer's and our models contain density inversions, but so do models for main-sequence stars.) Second, it is 
observationally (Osmer 1972) and theoretically (Wentzel 1970) likely that a density inversion will lead to turbulent motions that adjust the atmospheric structure appropriately rather than to drastic mass loss. Third, and finally, the observed masses, mass-loss rates, and even existence of red supergiants of high mass are in conflict with the idea of heavy mass loss from yellow-supergiant progenitors (Stothers 197.2b), quite apart from the problem of how a massive main-sequence star could have survived-its red and yellow pre-main-sequence stages.

\section{CONCLUSION}

Adoption of the Ledoux criterion for convective instability in calculating the evolution of a massive star causes the development of a shallow hydrogen gradient in the intermediate zone, which makes the envelope expand rapidly into the red-supergiant configuration during the early stages of core helium depletion. A blue loop on the $\mathrm{H}-\mathrm{R}$ diagram ensues if $\mathrm{Z}_{\mathrm{e}}, \mathrm{Y}_{\mathrm{e}}$, or the ${ }^{12} \mathrm{C}+\alpha$ reaction rate is sufficiently large, or if the $3 \alpha$ reaction rate is sufficiently small. It appears that these quantities are related, respectively, to the influence of opacity on the location of the base of the outer convection zone, to the mass of the core, and to the thermal properties of the core. The blue loops, however, are not 
particularly sensitive to the choices of $\mathrm{X}_{\mathrm{CN}^{\prime}} \alpha$, or low-temperature opacity. Our main results are summarized in table 3 . Whether the star crosses the Hertzsprung gap once or three times during core helium burning, all of these passages occur on the Kelvin time scale of the envelope (approximately). Before each passage, a slight pause or fluctuation of the luminosity always develops. Exhaustion of fuel at the center, and the consequent switchover to a purely gravitational source of energy in the core, is the reason for the luminosity perturbation before the first and third passages. Thermal instability of the envelope, and the consequent reduction of pressure on the core, accounts for the luminosity perturbation before the second passage. A general rule concerning the evolutionary tracks on the $\mathrm{H}-\mathrm{R}$ diagram is that the fine details become more obvious when $\mathrm{X}_{\mathrm{e}}$ or $\mathrm{Z}_{\mathrm{e}}$ is large.

Unfortunately, the blue-to-red ratio for supergiants of $\sim 15 \mathrm{M}_{\odot}$ cannot be predicted with very much precision until accurate knowledge is obtained of $\theta_{\alpha}^{2}$ and of the stellar opacity at temperatures near $10^{6} \mathrm{~K}$. At present, a rather small value of $\theta_{\alpha}^{2}(\sim 0.1)$ is expected to be correct (Barker 1971; Vidal, Shaviv, and Kozlovski 1971), but the stellar opacity is a more perplexing problem (especially near $10^{6} \mathrm{~K}$ ) since the relevant atomic physics as 
well as $Z_{e}$ and the constituents of $Z_{e}$ are not yet well enough known (Carson et al. 1968). Our present models for $15 \mathrm{M}_{\odot}$ indicate, tentatively, that a blue loop will occur for any reasonable initial chemical composition (providing that the new $3 \alpha$ rate is correct but regardless of the value of $\theta_{\alpha}^{2}$ ) and also that $\tau_{b}{ }^{/ T} \mathrm{He}_{\mathrm{e}}=0.5 \pm 0.2$.

A more precise statement can be made about the maximum effective temperature which is attained if the sequence has a blue loop. We find that this quantity is relatively invariant for our sequences at $15 \mathrm{M}_{\odot}$, which agree well, in this respect, with the two sequences obtained earlier by Stothers and Chin (1968) and by Robertson (1972). ${ }^{6}$ Thus, helium burning in blue supergiants of

${ }^{6}$ However, the maximum effective temperature attained is slightly cooler than average for the earlier sequence calculated by Stothers and Chin because of their specification of no chemical homogenization at all in the intermediate zone and their adoption of exaggerated lowtemperature opacities (given by Kramers law). The opacity effect alone can be seen explicity in our present work by comparing sequences $15-\mathrm{P}$ and $15-\mathrm{Q}$. On the other hand, Robertson's sequence becomes slightly hotter than average because of extensive chemical homogenization in the 
intermediate zone (due to a deep convective envelope on the red-supergiant branch).

$\sim 15 \mathrm{M}_{\odot}$ (based on the Ledoux criterion) is expected to occur within an effective temperature range from $\log \mathrm{T}_{\mathrm{e}} \approx 4.1$ to $\log \mathrm{T}_{\mathrm{e}}=$ $4.22 \pm 0.03$, since stabilization of the rapidly contracting envelope always first occurs when $\log \mathrm{T}_{\mathrm{e}} \approx 4.1 .^{7}$

$$
{ }^{7} \text { The effect of increasing the parameters } \theta_{\alpha}^{2} \text { or } \mathrm{Z}_{\mathrm{e}} \text {, }
$$

or of decreasing the $3 \alpha$ rate, is to increase only moderately $T_{b} / \tau_{\mathrm{He}}$ and $\log \mathrm{T}_{\mathrm{e}}$ at the tip of the blue loop. The corresponding effect of increasing $X_{e}$ or $X_{C N}$ is insignificant.

At $30 \mathrm{M}_{\odot}$, adoption of the Ledoux criterion does not yield a blue loop for any reasonable initial chemical composition. Unless convection in the intermediate zone (which we have ignored) penetrates to a deeper level than does the outer convection zone, the loop is unlikely to occur at this mass. 


\section{APPENDIX}

\section{THE STELLAR-EVOLUTION PROGRAM}

The stellar-evolution program developed for our work has been based on the standard method of numerical relaxation; the particular version of the method employed is that of Kippenhahn, Weigert, and Hofmeister (1967), with only minor modifications. We shall present here the details of the input physics that we have adopted because such information is useful for comparisons with other work, for assessing present limitations of the physics, and for justifying our various approximations.: The same program has already been used for the models in two recent papers of ours (Stothers 1972b; Stothers and Chin 1972).

\section{a) Thermodynamic State}

The thermodynamic state of the interior (in massive stars) is represented accurately by a mixture of nondegenerate gas and radiation. The state of the gas is computed for the following elements: hydrogen and helium in the stages $\mathrm{H}_{2}, \mathrm{H}, \mathrm{H}^{+}, \mathrm{He}, \mathrm{He}^{+}$, and $\mathrm{He}^{++}$; a hypothetical metal, with low ionization potential, in the stages $M$ and $\mathrm{M}^{+}$; and all other metals in the fully ionized state or the neutral state (depending, for simplicity, on whether 
helium is or is not completely ionized). The hypothetical metal is assumed to have the following atomic properties: $I=7.5 \mathrm{eV}$, $B(1) / B(0)=1.25, A=40$, and $X_{M}=0.08 Z_{e}$ (Demarque 1960; Iben 1963). The average atomic weight of all the other components of $\mathrm{Z}_{\mathrm{e}}$ is taken to be $\mathrm{A}=16$ (Traving 1966).

b) Opacity

Opacities calculated by Cox and Stewart (1965), including line absorption, have been adopted in the form given by Stothers and Simon (1970) as an improvement of Christy's (1966) formula, for temperatures higher than $4000^{\circ} \mathrm{K} . \mathrm{H}^{-}$opacities as given by Kippenhahn, Temesvary, and Biermann (1958) have been adopted for temperatures below $3000^{\circ} \mathrm{K}$. A temperature-weighted mean between the two sets of opacity has been employed for the intervening temperature range. Compton scattering by free electrons, in the form given by Deinzer and Salpeter (1965), has been adopted for temperatures higher than $8 \times 10^{7}{ }^{\circ} \mathrm{K}$.

Corrections to this prescription for opacity are undoubtedly needed, but the direction and amount of the total correction are still very uncertain. For example, compare the cited references in regard to the following quantities for opacity at high temperatures: 
the atomic model adopted (Carson, Mayers, and Stibbs 1968; Nikiforov and Uvarov 1970); the relative abundances of minor elements (Carson et al. 1968; Ezer and Cameron 1971); autoionizing states (Watson 1969a; Carson 1972); and electron correlations (Watson 1969b; Diesendorf 1970). Fortunately, in our models of massive stars, the densities are too small for electron correlations to be important. At low temperatures, however, we have omitted $\mathrm{H}_{2}, \mathrm{H}_{2} \mathrm{O}, \mathrm{CO}$, and $\mathrm{CN}$, which are probably the most important molecular sources of opacity; but, even so, for high masses these sources can influence the interior structure of the stellar envelope very little ( $($ IIIb; Auman and Bodenheimer 1967). Our only important omission seems to be absorption due to ${ }^{12} \mathrm{C}$ and ${ }^{16} \mathrm{O}$ in the dehydrogenized core, but the resulting formal error in opacity is $\leqslant 10$ percent (cf. Cox and Stewart 1965).

\section{c) Nuclear Reaction Rates}

The following nuclear processes have been taken into account: $4^{1} \mathrm{H} \rightarrow{ }^{4} \mathrm{He}\left(\mathrm{CN}\right.$ cycle), $3{ }^{4} \mathrm{He} \rightarrow{ }^{12} \mathrm{C}$, and ${ }^{12} \mathrm{C}+{ }^{4} \mathrm{He} \rightarrow{ }^{16} \mathrm{O}$. Energy-generation rates for the equilibrium reactions have been taken from Clayton (1968). For hydrogen burning, we have assumed that $\mathrm{x}_{\mathrm{CN}}=\frac{1}{3} \mathrm{z}_{\mathrm{e}}$ (Traving 1966) while ignoring the contribution of 16 o to the full CNO bi-cycle. The recently revised 300 rate (Austin, 
Trentelman, and Kashy 1971) is smaller than the rate quoted by Clayton by a factor $\exp \left(-1.38 / \mathrm{T}_{8}\right)$, and has been used in our latest model sequences. The rate for ${ }^{12} \mathrm{C}+\alpha$ is so uncertain (Barker 1971) that we have left as a free parameter $\theta_{\alpha}^{2}$, the reduced $\alpha$-particle width of the $7.12-\mathrm{MeV}$ level in ${ }^{16} \mathrm{O}$. The revised rate for ${ }^{14} \mathrm{~N}+\propto$ (Couch et al. 1972) indicates that nitrogen will be depleted only after the onset of the $3 \alpha$ reactions, and so ${ }^{14} N+a$ has been neglected as an energy-generation source. Electron-screening corrections to all the rates have been ignored as being unimportant because of the low densities encountered in massive stars.

\section{d) Convection}

Convection in the deep interior has been treated as fully adiabatic. In the outer envelope, nonadiabatic convection has been calculated according to the prescription given by Iben (1963, 1965), who adopted Böhm-Vitense's (1958) version of the mixing -length theory. Both the low-temperature opacity and the ratio of convective mixing length to density scale height have been left as adjustable parameters.

Convective overshooting at the inner boundary of the convective envelope has been calculated in an approximate way, which 
mimics a very thin semiconvective zone (Ledoux 1947; Chiosi and Summa 1970). At the outer boundary of the helium-burning convective core, overshooting has been neglected because of its already demonstrated insignificance in massive stars (Robertson 1972). All boundaries between radiative and convective zones have been fixed by requiring equality of the radiative and adiabatic temperature gradients on the convective sides of the boundaries (Härm and Schwarzschild 1955).

\section{e) Semiconvection}

Semiconvection has been ignored in our models even though it is occasionally implied by the Ledoux criterion for convective instability in the intermediate zone of varying hydrogen-helium content. Its presence at the inner boundary of the convective envelope (above) and at the outer boundary of the helium-burning convective core (Robertson 1972) has not been explicitly taken into account in our models, since, for massive stars, these semiconvective boundary layers are very thin. .

\section{f) Surface Boundary Conditions}

The uppermost layers of the atmospheres of our models have been assumed to be in radiative equilibrium. Eddington's 
(1926) approximate solution for a radiative atmosphere has been used for the local temperature structure, and an accurate integration of the equation of hydrostatic equilibrium has been performed for the pressure structure. The inward atmospheric integration is terminated either at the layer where the local temperature is equal to the effective temperature of the star or at the layer where convection begins (whichever comes first). The inward envelope integration is then begun as a smooth continuation from this point. It is terminated at mass fraction $q=0.90$, where the values of the physical variables are taken for use in the relaxation part of the program. 


\section{REFERENCES}

Auman, J. R., and Bodenheimer, P. 1967, Ap. J., 149, 641.

Austin, S. M., Trentelman, G. F., and Kashy, E. 1971 , Ap. J. (Letters), 163, L79.

Barbaro, G., Chiosi, C., and Nobili, L. 1971, in Colloquium on Supergiant Stars, ed. M. Hack (Trieste: Trieste Astronomical Observatory), p. 334 .

Barker, F. C. 1971, Australian J.Phys., 24, 777.

Bisnovatyi-Kogan, G. S., and Nadezhin, D. K. 1972, Ap. and Space Sci., 15, 353.

Böhm-Vitense, E. 1958, Zs.f. Ap., 46, 108.

Carson, T. R. 1972, unpublished.

Carson, T. R., Mayers, D. F., and Stibbs, D. W. N. 1968, M.N.R.A.S. , 140, 483 .

Chiosi, C., and Summa, C. 1970, Ap. and Soace Sci., 8, 478.

Christy, R. F. 1966, Ap. J., 144, 108.

Clayton, D. D. 1968, Principles of Stellar Evolution and Nucleosynthesis (New York: McGraw-Hill Book Company).

Couch, R. G., Spinka, H., Tombrello, T. A., and Weaver, T. A. 1972, Ap. J., 172, 395.

Cox, A. N., and Stewart, J. N. 1965, Ap. J. Suppl., 11, 22.

Dallaporta, N. 1971, in Colloguium on Supergiant Stars, ed. M. Hack, (Trieste: Trieste Astronomical Observatory), p. 359.

Deinzer, W., and Salpeter, E. E. 1965, Ap. J., 142, 813.

Demarque, P. 1960, Ap. J., 132, 366. 
Diesendorf, M. O. 1970, Nature, 227, 266.

Eddington, A. S. 1926, The Internal Constitution of the Stars (Cambridge: Cambridge University Press).

Ezer, D., and Cameron, A. G. W. 1971, Ap. and Space Sci., Ll, 52 .

Fricke, K., Stobie, R. S., and Strittmatter, P. A. 1971, M.N.R.A.S , 154,23 .

Fricke, K., and Strittmatter, P. A. 1972, M.N.R.A.S., 156, 129.

Härm, R., and Schwarzschild, M. 1955, Ap. J., 121, 445.

Hayashi, C., Hōshi, R., and Sugimoto, D. 1962, Progr. Theoret. Phys.- Suppl. (Ḱyoto), No. 22.

Henyey, L., Vardya, M. S., and Bodenheimer, P. 1965, Ap. J. 142,841 .

Hofmeister, E. 1967, Zs. f. Ap., 65, 164.

Hofmeister, E. Kippenhahn, R., and Weigert, A. 1964, Zs. f. Ap., 60,57 .

Iben, I., Jr. 1963, Ap. J., 138, 452.

- 1964, ibid., 140, 1631.

- 1965, ibid., 141, 993.

- 1966a, ibid., 143, 505.

- 1966ㅁ, ibid., p. 516 .

Kippenhahn, R., Meyer-Hofmeister, E., and Thomas, H. C. 1970, Astr. and Ap., 5, 155.

Kippenhahn, R., Temesvary, S., and Biermann, L. 1958, Zs. f. Ap., 46, 257. 
Kippenhahn, R., Weigert, A., and Hofmeister, E. 1967, in Methods in Computational Physics, ed. B. Alder (New York: Academic Press), Vol. 7, p. 129.

Kozlowski, M. 1971 , Ap. Letters, 9, 65.

Lauterborn, D., Refsdal, S., and Roth, M. L. 1971, Astr. and Ap., 13,119 .

Ledoux, P. 1947, Ap. J., 105, 305.

Meyer-Hofmeister, E. 1972, Astr. and Ap., 16, 282.

Nikiforov, A. F., and Uvarov, V. B. 1970, Dokl. Akad. Nalik SSSR (Ser. Mat. Fiz.) 191, 47.

Osmer, -P.-S. 1972, Ap. J. Suppl., 24, 255.

Paczynski, B. 1970a, Acta Astr. 20, 47. - 1970 b, ibid ; p. 195.

Robertson, J. W. 1972, preprint.

Sakashita, S., and Hayashi, C. 1961, Progr. Theoret. Phys. (Kyoto), 26, 942 .

Schwarzschild, M., and Härm, R. 1958, Ap. J., 128, 348.

Simon, N. R., and Stothers, R. 1970, Astr. and Ap. 6, 183.

Simpson, E. E. 1971, Ap. J., 165, 295.

Stein, R. F. 1966, in Stellar Evolution, eds. R. F. Stein and A. G. W. Cameron (New York: Plenum Press), p. 3.

Stothers, R. 1963, Ap. J., 138, 1074. . 1964, ibid., 140, 510. - 1966, ibid., 143, 91. 
. 1970, M.N.R.A.S., $151,65$.

- 1972a, Astr. and Ap., 18, 325.

. 1972b, Ap. J., in press.

- 1972c, Nature, in press.

Stothers, R., and Chin, C. -w. 1968, Ap. J., 152, 225.

. 1969, ibid., 158, 1039.

. 1972, ibid., in press.

Stothers, R., and Simon, N. R. 1970, Ap. J., 160, 1019.

Tanaka, Y., and Sakashita, S. 1964, Pub. A. S. Japan, 16, 173.

Traving, G. 1966, in Abundance Determinations in Stellar Spectra (IAU Symp. No. 26), ed. H. Hubenet (London: Academic Press), p. 213.

Vidal, N. V., Shaviv, G., and Kozlovsky, B. Z. 1971, Ap. Letters, 9,131 .

Watson, W. D. 1969a, Ap. J. 157, 375.

- 1969b, ibid., 158, 303.

Wentzel, D. G. 1970, Ap. J., 160, 373. 
TAB LE 1

PREVIOUS EVOLUTIONARY SEQUENCES OF MODELS

FOR MASSIVE STARS BASED ON THE

LEDOUX CRITERION

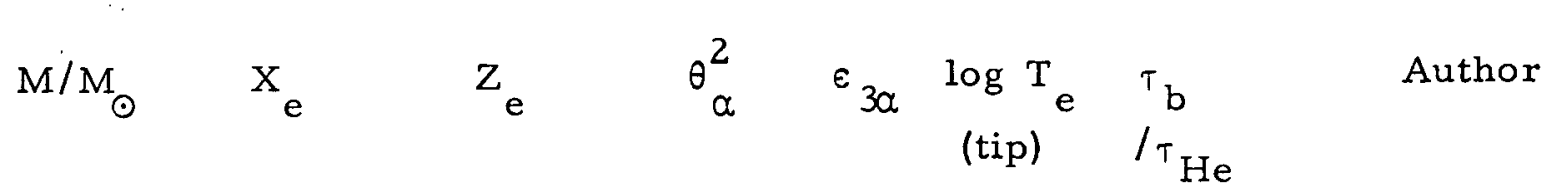

\begin{tabular}{|c|c|c|c|c|c|c|c|}
\hline $9^{*}$ & 0.739 & 0.021 & 0.100 & old & $\cdots$ & 0.0 & Hofmeister (1967) \\
\hline $9^{\dagger}$ & 0.602 & 0.044 & 0.100 & old & 4.03 & 0.5 & Hofmeister (1967) \\
\hline 10 & 0.700 & 0.030 & 0.085 & old & 4. 11 & 0.5 & Paczynski (1970a, b) \\
\hline 15 & 0.700 & 0.030 & 0.085 & old & $\ldots$. & 0.0 & Paczynski (1970a, 므) \\
\hline 15 & 0.700 & 0.030 & 0.100 & old & 4.17 & 0.6 & Stothers and Chin (1968) \\
\hline 15 & 0.602 & 0.044 & 0.100 & old & 4. 25 & 0.6 & Robertson (1972) \\
\hline 20 & 0.617 & 0.020 & 0.100 & old & $\cdots$ & $0.0^{\ddagger}$ & Robertson (1972) \\
\hline 20 & 0.602 & 0.044 & 0.060 & old & 4.27 & 0.8 & Chiosi and Summa (1970 \\
\hline 30 & 0.750 & 0.020 & 0.085 & old & $\ldots$ & 0.0 & Simpson (1971) \\
\hline
\end{tabular}

* Also calculated by Kippenhahn, Meyer-Hofmeister, and Thomas (1970) and by Meyer-Hofmeister (1972), with and without axial rotation.

† Also calculated by Lauterborn, Refsdal, and Roth (1971), by Fricke, Stobie, and Strittmatter (1971), and by Fricke and Strittmatter (1972).

\# This sequence was terminated when $\mathrm{Y}_{\mathrm{C}}=0.447$, but no blue loop had yet developed. 


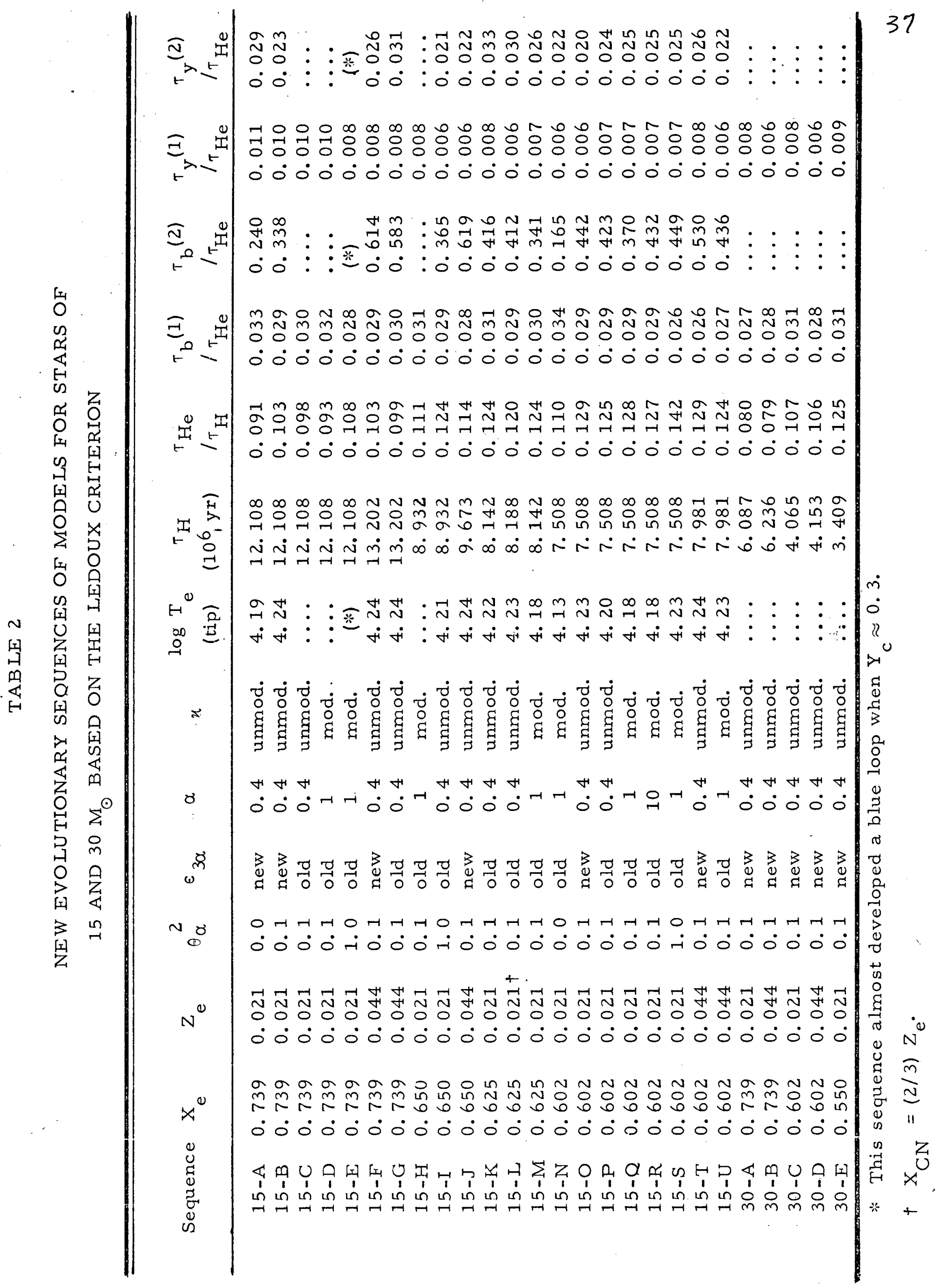


TAB LE 3

INPUT PARAMETERS FOR WHICH

A BLUE LOOP DEVELOPS

\begin{tabular}{ccccc}
\hline \hline $\mathrm{M} / \mathrm{M}_{\odot}$ & $\mathrm{X}_{\mathrm{e}}$ & $\mathrm{z}_{\mathrm{e}}$ & $\theta_{\alpha}^{2}$ & $\varepsilon_{3 \alpha}$ \\
\hline 15 & $\leqslant 0.62$ & 0.021 & 0.0 & old \\
15 & $\leqslant 0.64$ & 0.021 & 0.1 & old \\
15 & $\leqslant 0.70$ & 0.021 & 1.0 & old \\
15 & all & 0.044 & 0.1 & old \\
15 & all & all & all & new \\
30 & none & none & none & new or old \\
\hline
\end{tabular}

* An entry "all" or "none" refers to the following ranges:

$x_{e}=0.602-0.739, z_{e}=0.021-0.044$, and $\theta_{\alpha}^{2}=0.0-1.0$. 


\section{FIGURE CAPTIONS}

Fig. 1. - Theoretical evolutionary tracks in the H-R diagram for stars of 15 and $30 \mathrm{M}_{\odot}$. Tracks are labeled with the sequence numbers (see table 2), indicating the differences in initial chemical composition.

Fig. 2. - Hydrogen profiles for stellar models of $15 \mathrm{M}_{\odot}$ with $Z_{e}=0.021$ (solid lines, sequence 15-0) and $Z_{e}=0.044$ (dashed lines, sequence 15-F). Lettering index: a, end of core hydrogen burning; $\underline{b}$, tip of the red-supergiant branch; and $\underline{c}$, end of core helium burning.

Fig. 3. - Mass fractions of important composition boundaries as a function of $X_{e}$, for the sequences at $1 ; 5 M_{\odot}$ with $\mathrm{z}_{\mathrm{e}}=0.021$ (solid lines) and $\mathrm{z}_{\mathrm{e}}=0.044$ (dashed lines). Lettering index: a, maximum extent of the hydrogen-burning (ZAMS) convective core; $\underline{b}$, innermost extent of the outer convective envelope; $\underline{c}$, hydrogen shell peak at the end of core helium burning; d, hydrogen shell peak at the tip of the red-supergiant branch (during the early stages of core helium burning); and $\underline{e}$, maximum extent of the helium-burning convective core.

Fig. 4. - Mass fractions of important composition boundaries as a function of $\mathrm{X}_{\mathrm{e}}$, for the sequences at $30 \mathrm{M}_{\odot}$. Notation is the same as in figure 3 . 


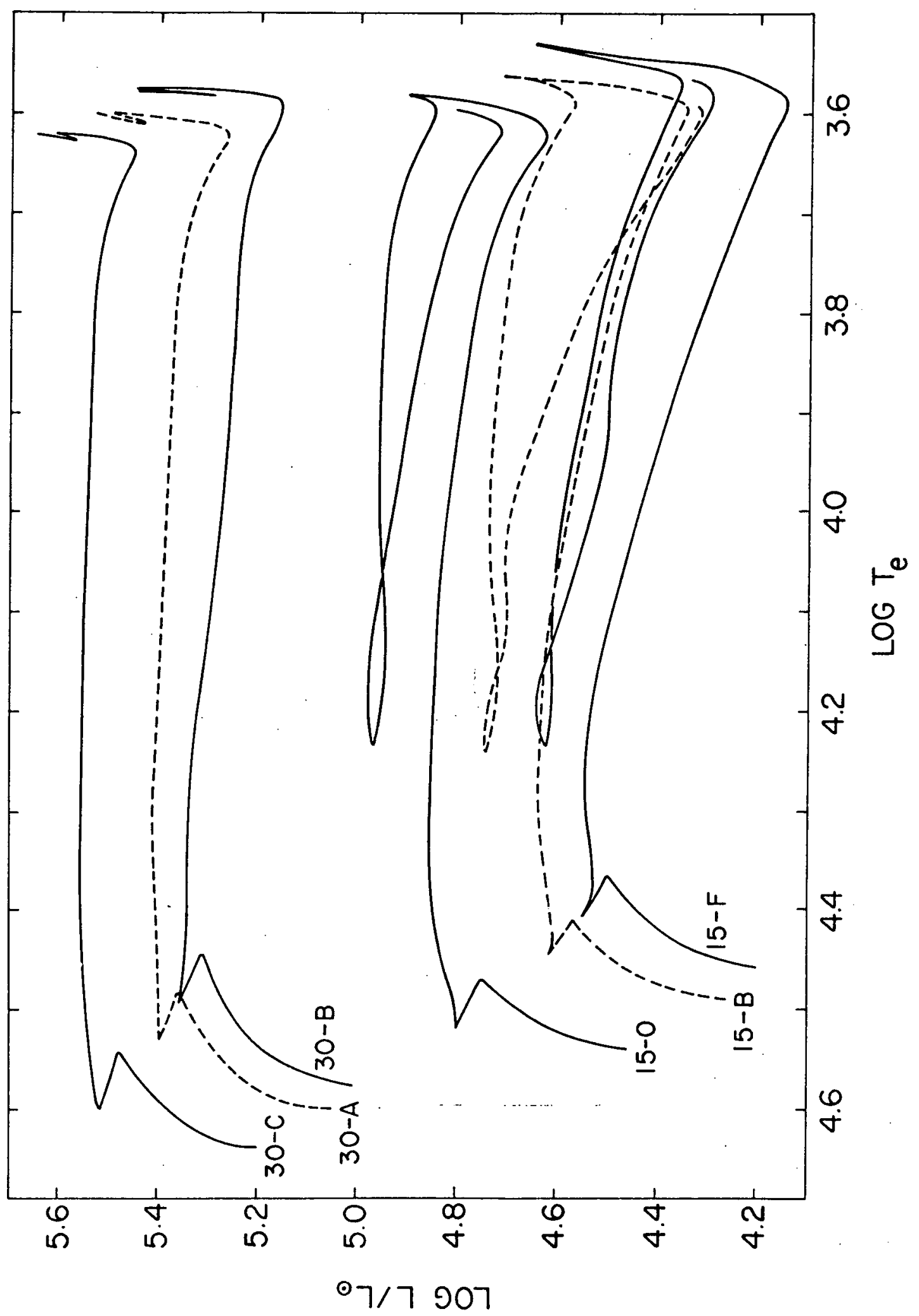




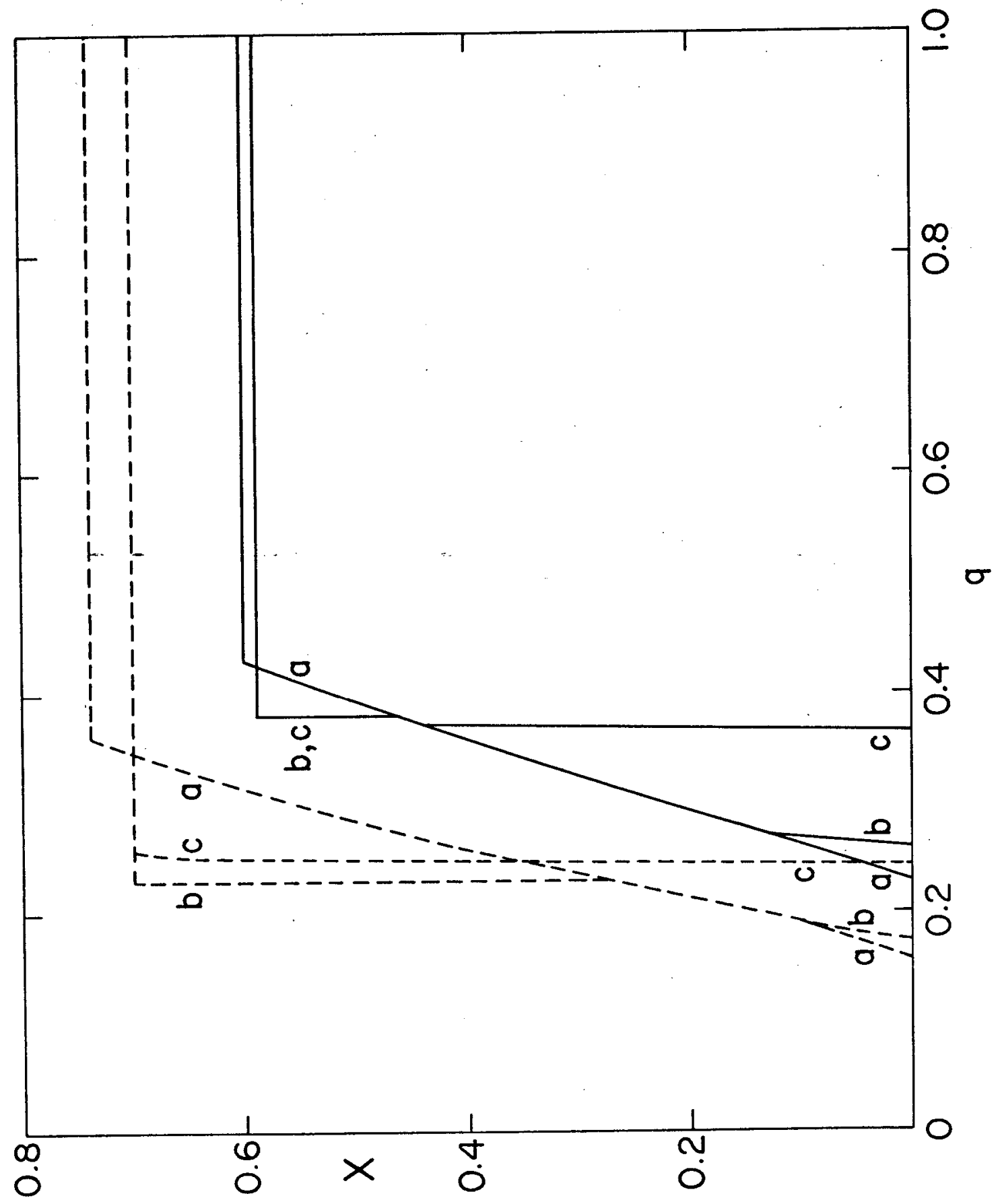




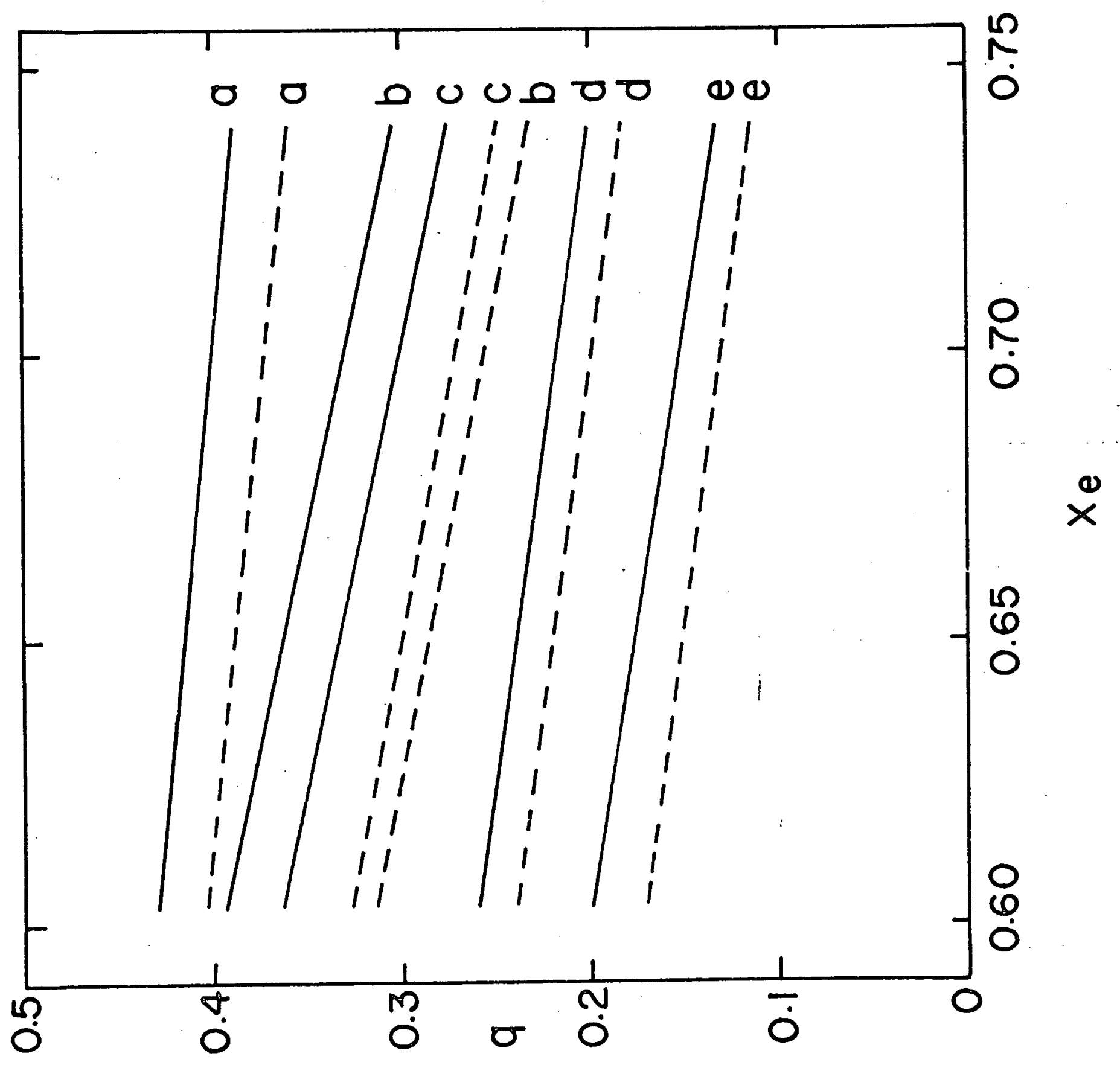

